\title{
Clinical Effect of Human Embryonic Stem Cells Therapy in Two Cases of Cerebral Palsy
}

\author{
Geeta Shroff
}

\begin{abstract}
Cerebral palsy (CP), a neurological disorder, has no known cure. The treatments with medicines, surgery and rehabilitation therapies may improve the child's condition but satisfactory outcome has not yet been achieved. Human embryonic stem cells (hESCs) therapy has been used for the treatment of neurodegenerative and neurological disorders. Here we report findings of two patients suffering from $\mathrm{CP}$, after undergoing treatment with hESC therapy. The treatment consisted of phases in which $0.25 \mathrm{~mL}$ ( $<4$ million cells) hESCs was administered through intramuscular route once daily and $1 \mathrm{~mL}$ of hESCs $(<16$ million cells) was administered through intravenous route twice every 7 days. A marked improvement in cognitive and motor functions was seen in the patients after the treatment.
\end{abstract}

Keywords: Human embryonic stem cells; Cerebral palsy; Neurological disorder; Intravenous route; Cognitive and motor functions

\section{Introduction}

Cerebral palsy (CP) is a neurological disorders caused by lack of oxygen to the brain due to early prenatal, perinatal or postnatal injury, structural abnormalities or infections in the brain [1]. This motor disability generally appears in infancy or early childhood [2].

There is no known cure for CP. Surgery, physiotherapy, occupational therapy or speech therapy may improve the child's condition but a satisfactory outcome has not yet been achieved [2].

In our previous studies, we have shown the improvement in the patient's condition suffering from $\mathrm{CP}$ [3] and cortical visual impairment after human embryonic stem cells (hESCs) therapy (paper published). Cell-based therapies are the potential interventions for perinatal brain injuries as they have the

Manuscript accepted for publication April 27, 2015

Nutech Mediworld, H-8, Green Park Extension, New Delhi 110016, India. Email: geetashroff@hotmail.com

doi: http://dx.doi.org/10.14740/jnr338e ability to protect or repair brain tissues [4]. hESC therapy may be used for the treatment of neurodegenerative and neurological disorders [5]. Here, we report our findings from two patients suffering from $\mathrm{CP}$, who had undergone treatment with hESC therapy.

\section{Case Reports}

A patented in-house (United States Granted Patent No. US8592,208,52) methodology was used for the isolation of hESCs that were used in the treatment of CP. The cell lines were obtained from a single, spare, throwaway pre-implantation stage fertilized ovum collected during in vitro fertilization (IVF) process. The details of the technique have been elaborated elsewhere [3]. The cell lines are free of animal product and are chromosomally stable. The evidence for the use of hESCs at Nutech Mediworld has been submitted and accepted at House of Lords, Regenerative Medicine, Science and Technology Committee [6].

A written informed consent was provided by the patients prior to start of the treatment. A detailed examination of the patients, radiological investigations with video graph recordings was done before and after the treatment. In-house physicians and nurses carefully observed all the patients for antigenic or paraphylactic responses.

\section{Case 1}

A 19-month-old male child was admitted at Nutech Mediworld on November 13, 2008 with complaints of delayed development milestone, poor trunk control, talking monosyllable and with spasticity of muscles. He had scoliosis. He "scooted" and was not able to crawl. The patient's mother had noticed delayed milestone in the baby (control of back after 12 months of age, unable to walk and stand, poor vocabulary). He could not walk properly and attained pincer grasp at 1 year. The patient was diagnosed with $\mathrm{CP}$ elsewhere. Hyperbaric oxygen therapy (HBOT) did not prove beneficial.

Physical examination revealed tightness in lower limb and elbow. His lower limbs muscles were weak. He experienced difficulty in sitting from lying position without support. Single photon emission computed tomography (SPECT) of brain 
Table 1. Motor System (Case 2)

\begin{tabular}{llllll}
\hline \multirow{2}{*}{ Parameters } & \multicolumn{2}{c}{ Upper limb } & & \multicolumn{2}{c}{ Lower limb } \\
\cline { 2 - 3 } \cline { 5 - 6 } & Right & Left & & Right & Left \\
\hline Tone & Hypotonic & Hypotonic & & Hypotonic & Hypotonic \\
Power & $3 / 5$ & $3 / 5$ & & $3 / 5$ & $3 / 5$ \\
Bulk & $\mathrm{N}$ & $\mathrm{N}$ & $\mathrm{N}$ & $\mathrm{N}$ \\
\hline
\end{tabular}

performed on November 12, 2008 revealed hypoperfusion in the basal ganglion, right inferior temporal lobe and right cerebellum. Gross motor function classification score (GMFCS) at baseline was 5 . There was no significant medical history, except for jaundice for 7 days after birth.

hESC therapy was given as a primary treatment along with rehabilitation. After the treatment that lasted for 310 days (therapy lasted 1,505 days), a marked improvement in cognitive and motor functions was seen including normal social behavior. SPECT scan showed moderate hypoperfusion in bilateral cerebral regions. His vocabulary improved, speech was normal and bowel/bladder control was also improved. He could sit and walk without support, climb stairs, kneel on sitting from quadripod position, hold objects properly and respond to all commands normally. GMFCS improved to 1 at the end of the therapy. He still has a slight impairment in his left hand.

\section{Case 2}

A 4.5-year-old female child was admitted on January 31, 2011 with complaints of delayed development, speech difficulty, impaired self help capabilities, impaired vision co-ordination and seizures. Her parents noticed slow development in child at 6 months. She was unable to trace light with her eyes and had started rolling over at age of 10 months and crawling at the age of 15 months. She was able to stand with support at 2 years. She was diagnosed with $\mathrm{CP}$ elsewhere.

Cystic changes were seen within bilateral parieto-occipital periventricular white matter that suggested the possibility of periventricular cystic leukomalacia. Electroencephalography (EEG) revealed focal interictal epileptiform abnormalities over the bilateral frontal regions. GMFCS at the baseline was 2. SPECT scan of brain done on January 20, 2011 disclosed abnormal cerebral perfusion scans with mild area of hypoperfusion in right parieto-temporal lobe. Analysis revealed that the patient was deficient of octadecanoylcarnitine enzyme.

On examination, we observed that the patient had speech difficulty, weak jaw movements and weak facial muscles. She was unable to hold pencil and write, had minimum eye contact and poor hand to eye co-ordination. She had intact bowel/bladder control and felt sensation but was unable to indicate. She had poor balance and could not walk without support. She had seizures since 2010 for which anticonvulsants was given. She was put on the regular sessions of physiotherapy.

Physical examination revealed bilateral divergent squint, flat foot with internal rotation of foot, pigeon chest, wide based gait and hypotonic limbs (Table 1). At 4 years, tongue tie was released surgically and she was put on valperin $(2.5 \mathrm{~mL})$ and levetiracetam $(2.5 \mathrm{~mL})$ twice daily for epilepsy.

At our facility, hESC was given as a primary treatment. After 642 days of the hESC therapy (including gap phases), she showed marked cognitive and motor function improvement like normal muscle power and tone, good eye contact, improved speech, grasped languages, good tripod pinch (able to write sentences), improved self help, good facial muscle strength and above normal intelligence. GMFCS was reduced to 1 at the end of the therapy. SPECT scan showed improvement in the degree of perfusion seen in the cerebral and cerebellar regions when compared to the previous SPECT scan.

\section{Discussion}

The present study provides an important novel direction for the use of hESC in treatment of patients with CP.

Treatment consisted of different phases with a gap phase of 4 - 6 months between the successive phases that allowed hESCs to grow, repair and regenerate. The duration of gap phase was arrived upon a concept of time taken for organogenesis (14 - 16 weeks) in human fetus [7].

There is no single treatment that is the best suited for CP [8]. Though natural recovery of $\mathrm{CP}$ is possible through rehabilitative therapies [9], Butler et al in their review were unable to conclude if neurodevelopment therapy was effective or ineffective in treating the symptoms of CP. They did not find any evidence for benefits of therapy in enhancing social-emotional, language, or cognitive domains of development [10]. In a recent review, botulinum toxin was found to be safe for the treatment of sialorrhea in children with $\mathrm{CP}$; however, the evidence to support this was quite low [11].

In the present study, in-house cultured hESCs created from a single fertilized ovum $24-48 \mathrm{~h}$ after fertilization was used to treat the patients. Human derived ESCs, derived primarily from in vitro fertilized embryos have shown promising early results [4]. Ma et al studied the effect of transplanted embryonic stem (ES) cell-derived cells in a 7-day-old hypoxic-ischemic encephalopathy (HIE) mouse model. ES cell-derived cells survived, migrated into the injury site, and specifically differentiated into neurons, thus showing improvement in learning ability and memory of the HIE mouse at 8 months post-transplantation [12]. Liu et al transplanted embryonic MSCs derived from hESCs in an ischemic rat. The cells migrated and differentiated into neuronal and endothelial cells, and improved behavioral functional outcome [13]. Mesenchymal stem cells (MSCs) have the ability to migrate to the injury site by communicating with other cells in the human body and 
exert clinical benefits [14]. Chemokines, cytokines and growth factors that are released upon injury act as migratory cues to the administered stem cells. These cues induce upregulation of selectins and activation of integrins on the stem cell surface that enables stem cells to interact, adhere and transmigrate through endothelium [15]. As the size of the hESCs used in our study was less than $1 \mu \mathrm{m}$, we could assume that these cells could have permeated through the parenchyma via blood brain barrier (BBB) and migrated in the affected regions. These cells then differentiated into neural cells and thus replaced the damaged cells. Homing of hESC to the injury site may provide an important clinical application of these cells in the future. SPECT scan was used as a prognostic tool for the detection of the extent of improvement in perfusion in the brain. A reduced tracer uptake is seen in the hypoperfused regions of the brain that is detected by the SPECT scan.

Our patients showed normal positive social behavior and improved muscle power after hESC infusion. We did not give any immune suppressants to our patients.

Though there is an ongoing debate for the use of hESC [4], their ability to cure the disease cannot be precluded. In our cases the cognition was better that allowed the patients with easy self care. In the present case series, after hESC therapy an improvement in the speech, vocabulary and memory was seen.

Due to the lack of effective interventions for neurodegenerative diseases, cell-based therapies carry future hope [4].

\section{Conclusion}

A marked cognitive and motor functions improvement was seen in CP patients after the hESCs therapy. Further research is necessary to elucidate the role of hESC in CP treatment.

\section{Acknowledgement}

The author acknowledges the parents, patients, doctors and staff of NTMW. The author also acknowledges Knowledge Isotopes Pvt. Ltd. (http://www.knowledgeisotopes.com) for the writing support.

\section{Conflict of Interest}

The authors do not have any conflict of interest.

\section{References}

1. Bathakur J. Human embryonic stem cell therapy, cer- ebral palsy, clinical satudy report. Published by Dr Geeta Shroff, Nutech Mediworld. 2012. ISBN No. 978-935067-565-6.

2. NIH, National Institute of Neurological Disorder and Stroke, Cerebral Palsy. Available at: <http://www.ninds. nih.gov/disorders/cerebral_palsy/cerebral_palsy.htm>. 2014.

3. Shroff G, Gupta A, Barthakur JK. Therapeutic potential of human embryonic stem cell transplantation in patients with cerebral palsy. J Transl Med. 2014;12(1):318.

4. Phillips AW, Johnston MV, Fatemi A. The potential for cell-based therapy in perinatal brain injuries. Transl Stroke Res. 2013;4(2):137-148.

5. Erceg S, Ronaghi M, Stojkovic M. Human embryonic stem cell differentiation toward regional specific neural precursors. Stem Cells. 2009;27(1):78-87.

6. House of Lords SATSC. Available at: <http://www.parliament.uk/documents/lords-committees/science-technology/RegenerativeMedicine/RegenMed.pdf $>$.

7. Medline Plus. Available at: <http://www.nlm.nih.gov/ medlineplus/ency/article/002398.htm>.

8. CDC; Centers for Disease Control and Prevention, Cerebral Palsy. Available at: < http://www.cdc.gov/ncbddd/ $\mathrm{cp} /$ facts.html>. 2013.

9. Damiano DL. Rehabilitative therapies in cerebral palsy: the good, the not as good, and the possible. J Child Neurol. 2009;24(9):1200-1204.

10. Butler C, Darrah J. Effects of neurodevelopmental treatment (NDT) for cerebral palsy: an AACPDM evidence report. Dev Med Child Neurol. 2001;43(11):778-790.

11. Porte M, Chaleat-Valayer E, Patte K, D'Anjou MC, Boulay C, Laffont I. Relevance of intraglandular injections of Botulinum toxin for the treatment of sialorrhea in children with cerebral palsy: a review. Eur J Paediatr Neurol. 2014;18(6):649-657.

12. Ma J, Wang Y, Yang J, Yang M, Chang KA, Zhang L, Jiang F, et al. Treatment of hypoxic-ischemic encephalopathy in mouse by transplantation of embryonic stem cell-derived cells. Neurochem Int. 2007;51(1):57-65.

13. Liu YP, Seckin H, Izci Y, Du ZW, Yan YP, Baskaya MK. Neuroprotective effects of mesenchymal stem cells derived from human embryonic stem cells in transient focal cerebral ischemia in rats. J Cereb Blood Flow Metab. 2009;29(4):780-791.

14. Kang SK, Shin IS, Ko MS, Jo JY, Ra JC. Journey of mesenchymal stem cells for homing: strategies to enhance efficacy and safety of stem cell therapy. Stem Cells Int. 2012;2012:342968.

15. Karp JM, Leng Teo GS. Mesenchymal stem cell homing: the devil is in the details. Cell Stem Cell. 2009;4(3):206216. 